\title{
Modelling and Performance of a Small Siphonic Hydropower System
}

\author{
B. H. Stark, E. Andò, G. Hartley
}

Faculty of Engineering, University of Bristol, Bristol BS8 1UB, United Kingdom

Email: bernard.stark@bristol.ac.uk

Bernard Stark

Electrical Energy Management Group

Dept. of Electrical \& Electronic Engineering

Merchant Venturers Building, Woodland Road, Bristol BS8 1UB, United Kingdom

$\mathrm{T} \quad+441179545499$

$\mathrm{F}+441179545206$

E bernard.stark@bristol.ac.uk

Keywords - Siphon turbine; system model; low-head hydropower; axial flow turbine; permanent magnet generator; rectifier;

Abstract - A siphon turbine is a low-head, axial-flow hydropower device that can be installed across an impound structure, such as a weir. A laboratory-scale siphon turbine and generator system is built from both off-the-shelf components and bespoke components designed using well documented guidance. This paper proposes a modelling method for the system, which allows performance to be assessed as a function of operating variables including head, electric load, runner speed and flow rate. An un-modified, commercially available propeller is used for the runner, whose blade geometry is defined within the model as a set of polynomials. This enables flow angles at any radial position on a blade to be determined as a function of the system operating point. Consequently, the impact of design changes on system performance can be quantified, such as part-load efficiency for example. Testing using the laboratory-scale system is conducted up to a maximum gross head of $0.25 \mathrm{~m}$, to validate the component models and then to demonstrate that the final system model provides satisfactory agreement with experiments over the operational speed and head range. The model therefore permits rapid conceptual design to be carried out without the need for computationally intensive co-optimisation of components.

\section{NOMENCLATURE}

\begin{tabular}{|c|c|}
\hline \multicolumn{2}{|c|}{ Electrical System } \\
\hline$i_{a}, i_{b}, i_{c}$ & Generator phase currents $[\mathrm{A}]$ \\
\hline$v_{a}, v_{b}, v_{c}$ & Generator phase voltages $[\mathrm{V}]$ \\
\hline$R_{a}$ & Generator phase resistance $[\Omega]$ \\
\hline$K_{e}$ & Machine back-EMF constant $\left[\mathrm{V}_{\mathrm{rms}} /(\mathrm{rad} / \mathrm{s})\right]$ \\
\hline$T_{g}$ & Generator electromagnetic torque $[\mathrm{Nm}]$ \\
\hline$\omega_{g}$ & Generator angular velocity $[\mathrm{rad} / \mathrm{s}]$ \\
\hline$\hat{V}_{e}, v_{e}$ & Back-EMF voltage (amplitude, instantaneous) [V] \\
\hline$I_{D C}, V_{D C}$ & DC load current and voltage $[\mathrm{A}, \mathrm{V}]$ \\
\hline$I_{S}, n$ & Diode dark current and ideality factor $[\mathrm{A},-]$ \\
\hline$V_{T}$ & Diode thermal voltage $[0.026 \mathrm{mV}]$ \\
\hline \multicolumn{2}{|c|}{ Mechanical system } \\
\hline$\omega$ & Runner angular velocity $[\mathrm{rad} / \mathrm{s}]$ \\
\hline$J_{\text {Total }}$ & Moment of inertia of shaft assembly $[\mathrm{Nm}]$ \\
\hline$T_{E u}$ & Euler torque $[\mathrm{Nm}]$ \\
\hline$T_{L}$ & Transmission loss torque $[\mathrm{Nm}]$ \\
\hline$G$ & Gear ratio of belt drive $[-]$ \\
\hline$H$ & Gross head across turbine (energy / unit weight) [m] \\
\hline$T_{r}$ & Runner shaft torque $[\mathrm{Nm}]$ \\
\hline$P_{L}$ & Power Loss $[\mathrm{W}]$ \\
\hline$P_{n}, h_{n}$ & Net power and Net head [m] \\
\hline$\xi, \lambda, \chi$ & Profile, incidence, and swirl loss coefficients \\
\hline$Q$ & Volume rate of flow through turbine system $\left[\mathrm{m}^{3} / \mathrm{s}\right]$ \\
\hline $\bar{A}_{d}$ & Mean ductwork area (weighted by length) $\left[\mathrm{m}^{2}\right]$ \\
\hline
\end{tabular}




$\begin{array}{cr}\rho & \text { Cold water density }\left[\mathrm{kg} / \mathrm{m}^{3}\right] \\ A_{r} & \text { Runner swept area }\left[\mathrm{m}^{2}\right] \\ U & \text { Runner velocity }[\mathrm{m} / \mathrm{s}] \\ i, \delta & \text { Angles of incidence and deviation }[\mathrm{rad}] \\ c_{i}, c_{o p} & \text { Incidence and over-prediction factors of } \\ \beta_{1}, \beta_{2} & \text { Entry and exit angles of blade measured to axial }[\mathrm{rad}] \\ \beta_{1 f}, \beta_{2 f} & \text { Apparent flow entry and exit angles to axial }[\mathrm{rad}] \\ \Delta \beta & \text { Blade camber angle } \beta_{2}-\beta_{1}[\mathrm{rad}] \\ l, b, t & \text { Blade profile chord, axial chord, thickness }[\mathrm{m}] \\ S & \text { Blade spacing from leading edge to leading edge }[\mathrm{m}] \\ V, W & \text { Absolute and relative flow velocities }[\mathrm{m} / \mathrm{s}] \\ \text { Runner-related subscripts } & \\ 1,2 & \text { Runner inlet and exit } \\ a, t & \text { Axial, tangential }\end{array}$

\section{INTRODUCTION}

Small-scale hydropower is a robust and comparatively environmentally benign power generation technology [1]. However, on this scale, many schemes incur disproportionately high capital costs per installed unit of power. A hydropower system using a siphonic ductwork configuration enables installation of the turbine across an existing impound structure, such as a weir, bypassing the requirement for a breach of the structure, see Figure 1. This could reduce the complexity of the civil works, which often account for a major proportion of capital costs of other forms of turbine installation. An advantage over more integrated technologies is that a comparatively small, replaceable, high-tech generation unit is combined with a comparatively large, low-tech and locally serviceable civil works component. Whilst a number of tethered drop-in, openflow concepts have been proposed, a system that constrains the flow can be preferable due to the increased power density and efficiency [2]. Therefore this specific installation arrangement has widespread potential. For example in England and Wales, the Environment Agency has identified nearly 26,000 existing impoundment structures or 'barriers' as providing an opportunity for hydropower development [3]. With national governments introducing so-called feed-in tariff schemes to encourage microgeneration, e.g. implemented by the UK government in April 2010 [4], the siphon turbine could satisfy an increasing demand for hydropower systems that are straightforward to install.

Figure 1: Schematic diagram of a siphon turbine installed over a weir with two paths for the water flow: over the weir and through the ductwork. The gross head is limited because the headwater can be raised only up to the weir height, the tailwater lowered only to the level of the exit orifice.

A siphon turbine, once primed, draws water up from a higher reservoir and discharges this water into a lower reservoir, as illustrated in Figure 1. The flow is driven by the difference in total head between the headwater and tailwater; 'head' referring to energy per unit weight of water and having meters as dimension. The shaft power is transmitted from the turbine through an airtight seal in the ductwork to the dry exterior where an electric generator converts it into electrical power. The turbine would typically process a pre-determined design flow rate, with the remainder overtopping the weir.

A portion of the theoretically available hydraulic power is lost through overcoming viscous forces within the flow field and at the boundaries, as well as in seal, bearing and mechanical transmission losses. Although not illustrated in Figure 1, the use of guidevanes immediately upstream of the runner, is essential for achieving high efficiencies [5, p.785], due to the reduction in draft tube swirl [6, p.636]. Guidevanes induce swirl immediately preceding the runner, such that the fluid momentum change occurring across the runner, results in a reduced tangential component of velocity in the draft tube, and hence reduced swirl loss. In addition, there are electric generator losses which are a function of the electric load.

In order to assess the power output performance of varying turbine designs, a mathematical model is required to rapidly quantify the influence of duct and turbine geometry on losses. Power output is also a function of the transmission, generator and power conditioning used. It is important to understand system behaviour when operational variables are not those for which a design has been optimised, particularly in small low-head installations, as it is possible that the envisaged operating conditions may not have been achieved [7]. Modelling this 'part-load' or 'off-design' behaviour therefore needs to take into account the often non-linear characteristics of each component of the system. The development of a complete siphon turbine model with these capabilities is the subject of this paper.

Albuquerque et al. [8] review the computational methods for hydropower design, citing methods that range from the design optimisation of turbomachinery using computational fluid dynamics, to simpler theoretical methods that are suitable for preliminary design phases but do not take into account blade geometry. It is valuable to be able to predict the off-design performance; that is, operation over a range of head and flow rate, not simply at design point. This allows increase in tolerance on site and installation options, and improves revenue predictions. The off-design performance is also important 
for the design of a power system and its control; for example automated start-up or, in the case of water being a valued commodity, variable-speed control. Singh and Nestmann [9] present a model that characterises the effect of blade exit angle on runner performance under part-load and deviation from the ideal flow rate, and emphasise the need for the development of validated models that characterise the influence of blade geometry.

In this paper, a complete parametric system model is developed, which relates the electrical output power to head and runner speed, whilst taking into account ductwork and blade geometry. In order to simplify the requirements of the mathematical model, guidevanes have been excluded from the system. This does of course negate the possibility of achieving the level of hydraulic efficiencies required for commercial applications; however, this is of secondary importance to the purpose of the paper, which is to present an uncomplicated demonstration of an accurate turbine model.

The structure of the paper is as follows. Section 2 describes the design of the experimental system, using well-documented empirical design rules, enabling model validation through testing. Section 3 develops the mathematical model of the system. Section 4 establishes the model parameters for the experimental system and validates the model at component level. Finally, Section 5 presents theoretical and experimental results for the system, and discusses the use of the mathematical model for system design and optimisation.

\section{EXPERIMENTAL SYSTEM}

The laboratory-scale device, shown in Figure 2, is designed for a 380mm weir, and installed within a flume $300 \mathrm{~mm}$ wide by $465 \mathrm{~mm}$ deep. In order to accurately measure flow rate through the turbine using the flume flow-meter, no water is allowed to flow over the top of the weir during testing.

Figure 2: Laboratory-scale system in flume with $0.02 \mathrm{~m}^{3} / \mathrm{s}$ capacity.

A flow contraction is designed to provide a maximum speed of approximately $2 \mathrm{~m} / \mathrm{s}$, a typical design value serving to minimise the probability of cavitation [10]. This, combined with the peak flow rate of the test facility, provides a runner housing diameter of $100 \mathrm{~mm}$, see the narrowest section labelled with $r_{D}=50$ in Figure 3 . After the runner, a $370 \mathrm{~mm}$ long diffuser or 'draft tube' increases flow area to an outlet with inner diameter of $177 \mathrm{~mm}$. This steadily decelerates the fluid, increasing the pressure and reducing energy losses. White [5, p.389] suggests that a diffusion angle between the draft tube wall and centreline of between $2.5^{\circ}$ and $7.5^{\circ}$ is desirable to minimise losses; Massey [6, p.213] quotes an optimum of $3^{\circ}$; Alexander et al. [10] quote an optimum of $4^{\circ}$, and report that, for their system, using $8^{\circ}$ incurs no loss in efficiency and brings the advantage of a shorter draft tube. An angle of $6^{\circ}$ is chosen here. Quantified design rules for ductwork are provided by Miller [11]. The tailwater is maintained at a sufficient level above the draft tube exit orifice to prevent air being ingested into the system and breaking the siphonic action. The resultant maximum difference in elevation between the headwater and tailwater is $0.28 \mathrm{~m}$. In order to minimise losses, the diameter of the ductwork shown in Figure 3 is chosen to be significantly larger than that of the runner section. The working length of the ductwork consists of a sharp protruding inlet preceding a straight section with inner diameter of $150 \mathrm{~mm}$. Following a segmented $88^{\circ}$ bend with a radius of curvature of $162 \mathrm{~mm}$, the contraction reduces the diameter to $100 \mathrm{~mm}$ within $57 \mathrm{~mm}$. The segmentation of the bend incurs a loss in efficiency [11, p.19] but brings manufacturing advantages.

Figure 3: Internal siphon geometry and 3D model. Dimensions in mm.

The narrowest section of the ductwork houses a propeller, shown in Figure 4. This rotating part, referred to as a 'runner', is the mechanism by which hydraulic power is converted to mechanical shaft power. The runner used for the laboratory-scale device is an un-modified, off-the-shelf propeller, which is both low cost and readily available. Consequently, its geometric design cannot be considered optimal for hydraulic efficiency.

Figure 4: Un-modified, off-the-shelf propeller used as the runner for the laboratory-scale device. Blade angles, relative to the primary axis, increase from around $45^{\circ}$ at the hub to $60^{\circ}$ at the tip. The blades are slightly cambered. Details of the exact geometry are provided in Figure 13 .

The runner shaft is supported at two points; firstly, by a bearing housing suspended by three stays immediately downstream of the runner; and secondly, by a sealed bearing where the shaft exits the ductwork. A belt drive increases the shaft speed of the generator by a ratio of 25:44. Two generators are used for experimentation; a 3-phase 8-pole permanent magnet outrunner machine (Turborix 832rpm/V CF2822) that has been modified to provide a higher voltage in conjunction with passive diode rectification (IR 21DQ04 2 Amp Shottky diodes), and a 12V brushed DC machine. Power is regulated using variable resistive loads. 


\section{PROPOSED MATHEMATICAL SYSTEM MODEL}

\subsection{Structure of the system model}

The aim of the system model presented here is to achieve broadly representative system behaviour with comparatively simple component models that facilitate component-by-component validation. The system model, illustrated in Figure 5, comprises six component models representing weir, ductwork, runner, transmission, generator and electric load. Runner and ductwork have been separated and will be validated individually. The transmission model incorporates the mechanical losses and inertia of the entire shaft assembly including belt transmission.

Figure 5: System model showing component input and output variables.

The change in velocity head between the headwater and tailwater is relatively small. Therefore, assuming hydrostatic pressure variation, the drop in total head across the turbine can be approximated by the difference in surface elevation between the headwater and tailwater, or 'gross head' $H$. The weir model defines the gross head, which is a function of the geometry of civil works and, is here, a weak function of the river flow rate. The power drop across the turbine can be approximated by the product of the gross head and the weight of water flowing through the ductwork per unit time.

The ductwork model calculates the flow rate $Q$ through the ductwork from the driving gross head, reduced by the net head $h_{n}$. The runner model calculates the net head resulting from a flow rate $Q$ and a runner speed $\omega$. This is done by summing runner associated losses and the useful power delivered to the shaft through the runner torque $T_{r}$. The runner, transmission loss, and generator torques provide the runner shaft angular velocity $\omega$ and generator speed $\omega_{g}$ via a transmission model. The generator and rectifier model converts speed into rectified generator output voltage $V_{D C}$ and electromagnetic generator shaft torque $T_{g}$ as a function of current $I_{D C}$ drawn by the load. The electric load model determines the current $I_{D C}$ drawn from the generator for a given supply voltage $V_{D C}$.

The input and output variables of each of the component models have been chosen so that adjacent physical components have meaningful input and output characteristics, see Figure 6. The runner's output characteristic, for example, is the torque/speed relationship as a function of flow rate, and the generator's input characteristic is the torque/speed relationship as a function of load current.

Figure 6: Schematic representation of the measurable input and output characteristics of the component models, with rough trends indicated.

\subsection{Electric load model}

The system model is developed here 'bottom-up' from the electric load, as this incorporates the main design constraint, namely the delivery of a minimum constant or time-varying electrical power. The load model represents an electrical system that is supplied via a regulated switched-mode power supply, which is the typical way of maintaining a constant voltage for electrical systems over a wide supply voltage range, usually at a high efficiency $\eta$ exceeding $90 \%$. Therefore the load supply current $I_{D C}(t)$ varies inversely with the supply voltage $V_{D C}(t)$, and proportionally to the power $P_{\text {Load }}(t)$ required by the load. The subscript $D C$ refers to direct current.

$$
I_{D C}=P_{\text {Converter }} \cdot \frac{1}{V_{D C}}=\frac{P_{\text {Load }}}{\eta_{\text {Converter }}} \cdot \frac{1}{V_{D C}} .
$$

The power drawn by the load under constant converter output voltage is independent of the hydropower system, as long as the supply voltage $V_{D C}$ is in the permitted range. As a first approximation, the power drawn by the load and efficiency of the converter are assumed to be constant. Further refinement of the load model could include a dependency of the efficiency on the output current and a variation of the load power represented by a sample time-series data set.

\subsection{Generator and rectifier model}

The load shares an electrical interface with the generator, assumed to be a permanent magnet generator with passive diode rectifier, see Figure 7. The model developed here can be applied to brushed DC machines, as shown later in section 4 . Lowcost induction machines are not considered, nor is active rectification using an inverter, which could increase system efficiency.

Figure 7: The generator and rectifier model represents a rectified 3-phase permanent magnet generator. The equivalent circuit diagram and time-domain voltage traces are shown here, see text for details.

The amplitude $\widehat{V}_{e}$ of the alternating back-EMF, that is the voltage induced in each phase of a permanent magnet generator, is proportional to generator speed $\omega_{g}$, and independent of load current (within a typical operating range) [12, p.63], 


$$
\widehat{V}_{e}=\sqrt{2} K_{e} \omega_{g} .
$$

Here, $K_{e}$ is the machine-dependant back-EMF constant expressed in units of $\mathrm{rms} \mathrm{V}$ per rad/s, and $\hat{V}_{e}$ denotes the amplitude of the time dependent, sinusoidal, back-EMF voltage $v_{e}(t)$. The back-EMF of phase $a$ is

$$
v_{e a}(t)=\widehat{V}_{e} \sin \left(\omega_{g} t+\varphi_{a}\right),
$$

where $\varphi_{a}$ is the phase angle at time $t=0$. The rectified output voltage $V_{D C}$ of a star-connected generator is $\sqrt{3}$ times the back-EMF minus a number of voltage losses, three of which are typically estimated in textbooks (e.g. [13, p.95]), namely those of the series inductance $L_{S}$, series resistance $R_{a}$ and the diodes, providing

$$
V_{D C}=\sqrt{3} \widehat{V}_{e}-V_{L S}-2 V_{R a}-2 V_{D} \text {. }
$$

Here, the simplifying assumption is made that that the DC load current $I_{D C}$ is large relative to the smoothing capacitor shown in Figure 7. This allows us to approximate the load current as constant, and therefore the phase $a$ current as a rectangular pulse train, illustrated in Figure 7, formed by the current of two diodes connected to phase $a$. This approach neglects the variation in phase currents due to charging and discharging of the filter capacitor during the rectification. It also makes the simplifying assumption that the machine's efficiency is not adversely affected by non-sinusoidal (but periodic) current draw.

The voltage drop $V_{L S}$ due to series inductance is a function of the phase frequency [13, p.108],

$$
V_{L S}=\frac{3}{\pi} \omega_{g} L_{S} I_{D C}
$$

The ohmic voltage drop is

$$
V_{R a}=R_{a} I_{D C}
$$

where $R_{a}$ is the ohmic resistance of a phase winding.

The diode forward voltage drop $V_{D}$ is determined using Shockley's simplified diode equation

$$
I_{D C}=I_{S} \mathrm{e}^{\left(\frac{V_{D}}{n V_{T}}\right)} .
$$

Here, $I_{S}$ and $n$ are device-specific constants and $V_{T}$ is the thermal voltage $0.026 \mathrm{mV}$.

On substituting equations (2.1), (2.4), (2.5), and (2.6) into (2.3), for $t=0$, we obtain an approximate model for the rectified generator

$$
\begin{aligned}
V_{D C}= & \sqrt{6} K_{e} \omega_{g}-\frac{3}{\pi} \omega_{g} L_{S} I_{D C} \\
& -2 R_{a} I_{D C}-2 \ln \left(\frac{I_{D C}}{I_{S}}\right) n V_{T} .
\end{aligned}
$$

Note that any temperature-induced reduction of magnetic field strength of permanent magnets, and thus of $K_{e}$, has been neglected.

The electromagnetic torque [12] developed by one phase of a synchronous generator is

$$
T_{a}(t)=\frac{v_{e a}(t) i_{a}(t) \cos (\varphi)}{\omega_{g}}
$$

where $\cos (\varphi)$ is the phase angle between the back-EMF voltage $v_{e a}$ and phase current $i_{a}$. Under the idealised assumptions of the phase currents being equal to the load current $I_{D C}$, and using equations (2.1) and (2.2), the phase $a$ torque $T_{a}$ is obtained. This is averaged over the conduction period, and multiplied by 2 as two phases provide torque at any one time,

$$
T_{g}=2 \cdot \frac{3}{2 \pi} \frac{\int_{\omega_{g} t=30^{\circ}}^{150^{\circ}} v_{e a} I_{D C} d\left(\omega_{g} t\right)}{\omega_{g}}=\frac{3 \sqrt{6}}{2 \pi} K_{e} \cdot I_{D C},
$$

thus providing the average electric torque of a passively rectified 3-phase permanent magnet generator. In further considerations we assume the generator torque $T_{g}$ to be constant and equal to this average, for a given load current.

\subsection{Transmission and inertia model}

The inertia model derives runner shaft speed $\omega$ and generator shaft speed $\omega_{g}=G \cdot \omega$ by integrating the angular acceleration $\dot{\omega}$. The angular acceleration is determined from the sum of torques acting on a rotating mass with a moment of inertia $J_{\text {Total }}$ 
that represents the generator, shaft, runner, belt drive and couplings. The inertia of the fluid is neglected. The angular acceleration is thus

$$
\dot{\omega}=\frac{1}{J_{\text {Total }}} \cdot\left(T_{r}-T_{L}-G \cdot T_{g}\right),
$$

where $T_{r}$ is the driving runner torque to be calculated by the runner model, and $T_{g}$ is the electric torque applied by the generator, transferred to the runner side by multiplying by the belt drive ratio $G . T_{L}$ is the mechanical transmission loss torque of bearings and belt drive. Belt drive loss torque has been shown experimentally in [14] to be a relatively weak function of load torque and speed, and to display a significant offset, whereas the efficiency is a strong function of these variables. Bearing loss torques are sensitive to non-radial loading, which can increase with load torque. Therefore, rather than using a constant efficiency model where $T_{L}=T_{r} \cdot(1-\eta)$, the transmission loss torque is approximated by a linear dependency on load torque and speed,

$$
T_{L}=T_{0}+C_{T} \cdot\left(G \cdot T_{g}\right)+C_{\omega} \cdot \omega .
$$

with coefficients $C_{T}$ and $C_{\omega}$ to be determined experimentally.

\subsection{Runner model (Axial-flow turbine)}

The specific speed $N_{S}$ of a turbine is widely used as a descriptor for pumps and turbines, and is given by

$$
N_{S}=\frac{N \cdot(P)^{\frac{1}{2}}}{h_{n}^{\frac{5}{4}}} .
$$

Here, $N$ is the shaft speed in rev/min, $P$ is the shaft output power in $\mathrm{kW}$, and $h_{n}$ is the net head of the turbine. The specific speed $N_{S}$ describes the speed at which a geometrically similar system that has been scaled to a $1 \mathrm{~m}$ head would need to operate, in order to generate $1 \mathrm{~kW}$ of shaft power. Quoted specific speed ranges, e.g. [10] and [16, p.685], for axial-flow turbines fall between 175 and $750 \mathrm{rev} / \mathrm{min}$. Typically, on having determined a required specific speed, the engineer selects a turbine design that is reported to perform at this specific speed. By contrast, the turbine model developed in the following sections does not rely on reported designs. It will provide the net head, shaft power and speed (and thus specific speed) directly from gross head $H$ and runner geometry, thus allowing, for example, the evaluation of new runner blade designs. The model will also allow a direct interface between the turbine properties and the electromechanical output.

\subsubsection{Model structure}

The runner model, illustrated in Figure 8, determines the runner net head $h_{n}$ and the runner torque $T_{r}$ as a function of flow rate $Q$ and runner speed $\omega$.

Figure 8: Runner model, represented as a block diagram showing component input and output variables.

Net head $h_{n}$ is the gross head $H$ reduced by the ductwork head loss $h_{\text {duct }}$ and represents the head available to the runner. Without the runner in-situ, the flow rate would accelerate until the increasing ductwork head loss was equal to the gross driving head. With the runner in-situ, the net head is apportioned to runner associated loss modes and a remaining component that provides useful runner torque, according to the runner geometry and the operating conditions.

Multiplying net head by the weight of water flowing through the turbine per unit time gives the net power available to the runner $P_{n}$, which is modelled as the sum of runner-associated losses and Euler power $P_{E u}$ (see section 3.5.4):

$$
h_{n} \cdot \rho g Q=P_{n}=\left(P_{\text {Lprof }}+P_{\text {Linc }}+P_{\text {Lswirl }}\right)+P_{E u},
$$

where

$$
P_{E u}=T_{E u} \cdot \omega .
$$

Runner-associated losses in axial flow turbines can be considered as broadly represented by the following three groups [8]:

1) Profile loss $P_{\text {Lprof }}$ as defined by Horlock $[15, \mathrm{p} .85]$ groups together losses owing to boundary layer growth along the runner blade and runner housing, flow separation at runner exit and tip losses.

2) Incidence or shock loss $P_{\text {Linc }}$ accounts for off-design operation, where the relative inlet flow velocity is not aligned with the blade entry angle $\beta_{1}$. Consequently the flow does not enter the blade passages smoothly, but strikes either the front or the back blade surfaces. This results in considerable eddy formation and dissipation of energy [6, p.651]. At the design speed and flow rate, incidence loss is zero if the blade has the appropriate twist. 
3) Swirl loss $P_{\text {Lswirl }}$ accounts for the tangential component of velocity induced by pre-swirl devices and the runner. This component increases the absolute velocity of the fluid and therefore the power lost in overcoming viscous forces within the flow field and at the boundary. Here there are no guidevanes or scroll-case to induce pre-swirl and swirl losses are considered to occur only downstream of the runner.

$P_{\text {Lprof }}, P_{\text {Linc }}$, and $P_{\text {Lswirl }}$, as well as Euler torque $T_{E u}$, remain to be derived as functions of $Q$ and $\omega$, in order to obtain the runner model outputs illustrated in Figure 8.

\subsubsection{Flow assumptions}

In what follows, the subscript 1 represents quantities evaluated at the point of runner entry, or inlet, and the subscript 2 represents those at the point of exit. Subscripts $a$ and $t$ represent axial and tangential flow components respectively. $V$ denotes absolute velocity, whereas $W$ and $\beta$ denote velocity and angle relative to the blade. All flow and runner associated velocities and geometric quantities are functions of radial position $r$, and are defined in Figure 9.

Figure 9: Cross-section of an individual blade showing velocity triangles and notation used for runner model.

The blades of a hydraulic turbine constrained by a tubular conduit can be modelled as a cascade that itself bounds the flow [16, p.647], especially for turbines of a high 'solidity', or chord-to-pitch ratio $l / s$. For very low solidity turbines, an alternative is to treat the individual blades as bodies in an external flow, as might be typical for a wind turbine. Axial-flow hydraulic turbines typically have a high solidity of 1 or higher, therefore the cascade method is adopted here. The runner shown in Figure 4 has a medium solidity of 0.72 at $\frac{2}{3}$ of the blade length.

To simplify the modelling procedure, the entry flow velocity $V_{1}$ is assumed to be axial and constant across the area $A_{d}$ of the runner housing minus the hub area, with no tangential component of velocity. This implies undeveloped flow, and a slip condition at flow boundaries. Conservation of mass and the assumption of incompressible flow imply that the axial component of velocity $V_{a}$ is maintained throughout the runner housing, thus

$$
V_{2 a}=V_{1 a}=V_{1}=V_{a}=\frac{Q}{A_{d}},
$$

where $Q$ is the volume rate of flow through the ductwork.

\subsubsection{Incidence, deviation and flow turning angle}

Let $U(r)$ represent the tangential velocity of a blade at the radial position $r$, and $\omega$ the angular velocity of the runner, thus

$$
U=r \omega .
$$

From basic trigonometry it follows that the flow strikes the blade at an angle

$$
\beta_{1 f}=\arctan \left(\frac{U}{V_{a}}\right)=\arctan \left(\frac{r \omega A_{d}}{Q}\right),
$$

thus at an angle of incidence

$$
i=\beta_{1}-\beta_{1 f},
$$

where $\beta_{1}$ is the blade entry angle. The blade redirects the flow so that the relative flow $W_{2}$ exits the blade at an angle $\beta_{2 f}$. Because there are a finite number of blades, this flow angle will be less than the blade angle by a value called the deviation $\delta$ $[17]$,

$$
\beta_{2 f}=\beta_{2}-\delta \text {. }
$$

Horlock [15, p.80] quotes Carter and Hughes, who developed a deviation model for 'low-speed' flow through gas turbine cascades with near zero incidence:

$$
\delta_{C \& H}=M\left(\frac{S}{l}\right)^{\frac{1}{2}} \cdot \Delta \beta .
$$

This formula shows deviation increasing with camber angle $\Delta \beta=\beta_{2}-\beta_{1}$ and decreasing with turbine solidity $l / s$. Albuquerque et al. [8] apply the above equation to design point operation of an incompressible flow hydraulic turbine, and, by matching experimental data, derive a necessary over-prediction factor $c_{o p}$ of 1.5. Since the runner model described here aims to predict off-design performance, an additional adjustment of the original equation is proposed. As the system moves away from the desired operating point, the relative velocity vector at the runner inlet develops a significant angle of incidence, therefore increasing the turning angle of the flow, which is assumed to increase the deviation as follows: 


$$
\delta=c_{o p} M\left(\frac{S}{l}\right)^{\frac{1}{2}}\left(c_{i} i+\left(\beta_{2}-\beta_{1}\right)\right) .
$$

For a physical interpretation of the summation of incidence and camber angle, the leading edge of the runner can be thought of as changing to towards the direction of flow at inlet, where the use of equation (4.9) is applicable; the validity of this approach is improved by the fact that the original experimental data used by Carter and Hughes [15, p.80] were derived from tests on on high camber blades [8].

Qui et al. [17] provide a graph of the factor $M$ against stagger angle $\theta=\frac{1}{2}\left(\beta_{2}+\beta_{1}\right)$, which can be approximated by

$$
M=0.041 \cdot \theta^{2}-0.0716 \cdot \theta+0.22 \text {. }
$$

Thus the full turning angle $\epsilon(r)$ of the fluid is now defined and can be written as

$$
\epsilon=i+\left(\beta_{2}-\beta_{1}\right)-\delta,
$$

thereby describing the onset of rotation of the fluid, which in turn requires the presence of torque.

\subsubsection{Runner shaft torque}

An expression for torque exerted by the fluid on the runner is sometimes referred to as the Euler torque equation, and the derivation can be found in many fluid mechanics texts, e.g. [6, p.633], [16, p.642], and [18, p.651]. The premise of the equation is that the torque exerted on the fluid is equal to the rate of increase of angular momentum of the fluid between the inlet and outlet of the runner and, assuming that there are no shear forces at these stations with moments about the runner axis, then this torque is also exerted by the fluid on the runner [6]. For zero tangential velocity at the runner inlet, the Euler torque is given by

$$
T_{E u}=\int_{A_{r}} V_{2 t} r d \dot{m}
$$

where $V_{2 t}$ is the tangential velocity component of the exit flow, $A_{r}$ is the runner swept area and $d \dot{m}$ the mass flow rate in $\mathrm{kg} / \mathrm{s}$ through an area $d A_{r}$ at a radial position $r$. It is important to note that although losses due to turbulence and viscous forces acting within the blade passages affect the tangential velocity field at the exit, they do not undermine the validity of equation (4.13) [6, p.633].

The tangential component of the exit flow $V_{2 t}$ needs to be adjusted to allow for deviation and can be obtained from the basic trigonometry

$$
\tan \left(\beta_{2}-\delta\right)=\frac{U-V_{2 t}}{V_{a}}
$$

Assuming axi-symmetric flow (cylindrical stream surfaces), the mass flow rate is $d \dot{m}=2 \pi r \cdot d r \cdot \rho \cdot V_{a}$, where $\rho$ is the density of the fluid. The Euler torque equation (4.13) thus becomes

$$
T_{E u}=2 \pi \rho \frac{Q}{A_{d}} \int_{r_{\text {hub }}}^{r_{\text {tip }}}\left[r \omega-\frac{Q}{A_{d}} \tan \left(\beta_{2}-\delta\right)\right] r^{2} d r,
$$

which can be evaluated for flow rates $Q$ and runner speeds $\omega$.

Opposing this torque are the previously mentioned loss mechanisms. These would prevent a free-running turbine, one with no opposing shaft torque, from spinning up to a speed where $T_{E u}=0$. In section 3.5.9, expressions for profile drag torque $T_{\text {Lprof }}$ and torque due to incidence $T_{\text {Linc }}$ are derived. The swirl loss mechanism is assumed to occur downstream of the runner and, neglecting fluid shear forces in a cross-sectional surface immediately downstream of the runner, swirl can be assumed not to provide a drag component on the runner. The runner torque $T_{r}$ is then

$$
T_{r}=T_{E u}-T_{\text {Lprof }}-T_{\text {Linc }},
$$

from which the runner shaft power $P_{r}$ can be calculated using

$$
P_{r}=T_{r} \cdot \omega
$$

\subsubsection{Specific profile energy loss}

Specific energy loss has dimensions of Joules per unit mass of fluid. The specific profile energy loss can be modelled as being proportional to the square of the exit flow velocity $W_{2}$ relative to the runner [8], 


$$
Y_{\text {Lprof }}=\frac{1}{2} \xi\left(W_{2}\right)^{2} .
$$

Using the velocity triangles of Figure 9 and assuming that the axial flow velocity is constant at all locations within the runner housing, the velocity $W_{2}$ is

$$
W_{2}=\frac{Q}{A_{d}} \cdot \frac{1}{\cos \left(\beta_{2}-\delta\right)}
$$

and therefore independent of runner speed. Soderberg (in [15, p.87]) provides a profile loss coefficient $\xi$, through correlation of specific profile energy loss against a number of non-dimensional groups:

$$
\xi=\left(\frac{10^{5}}{R e}\right)^{\frac{1}{4}} \cdot\left(\left(1+\xi_{1}\right)\left(0.975+0.075 \frac{b}{B}\right)-1\right)
$$

Here, $b$ is the axial chord, and $B$ the blade length. $R e$ is the Renolds number

$$
R e=\frac{\rho W_{2} D_{h}}{\mu}
$$

based on the throat hydraulic mean radius

$$
D_{h}=\frac{2 B s \cos \left(\beta_{2 f}\right)}{s \cos \left(\beta_{2 f}\right)+B},
$$

where $s$ is the blade spacing, and $\mu$ the viscosity. Finally, $\xi_{1}$ is provided graphically in [15, p.87] as a function of the flow turning angle $\epsilon$ (equation (4.12). The polynomials

$$
\xi_{1}=\xi_{0}+0.0024 \cdot \epsilon+0.0215 \cdot \epsilon^{2}
$$

and

$$
\xi_{0}=0.071-0.19 \cdot \frac{t}{l}+0.27 \cdot\left(\frac{t}{l}\right)^{2}
$$

provide a good approximation within the ranges plotted, where $t / l$ is the blade thickness to chord ratio.

The original experimental data used for Soderberg's correlation was derived from gas turbine tests under small deviation and zero incidence angle $\left(\beta_{1 f}=\beta_{1}\right)$. Horlock [15, p.87] states that reasonable accuracies are achieved over a wide range of Reynolds numbers, and that due to the use of experimental data, additional correction for compressibility is not required for high-speed gas turbines. However, the absolute accuracy of application with incompressible-flow hydraulic turbines is unclear. Furthermore, error can be expected if blade twist does not match the relative velocity at entry at all radial stations, which is compounded when the ratio of flow rate to runner speed moves off design point. However, this paper follows the precedent of Albuquerque et al. [8] in using this equation as part of the wider model, for its use in demonstrating the correct trends.

On substituting equations (4.19) - (4.24) into (4.18), we obtain a fully defined expression for the profile loss that is approximately proportional to $Q^{5 / 2}$ and only a weak function of runner angular velocity $\omega$.

\subsubsection{Specific incidence energy loss}

Specific incidence energy loss $[15, \mathrm{p} .78][8]$, also known as shock loss, is proportional to the square of $W_{\text {inc }}$, which is the difference between the runner velocity $U$ and the tangential component of velocity of flow entering the runner that would satisfy zero incidence, Figure 9.

$$
Y_{\text {Linc }}=\frac{1}{2} \lambda\left(W_{i n c}\right)^{2}
$$

Basic trigonometry and substitution of equation (4.5) gives

$$
Y_{\text {Linc }}=\frac{1}{2} \lambda\left(\frac{Q}{A_{d}} \cdot \tan \left(\beta_{1}\right)-r \omega\right)^{2},
$$

showing specific incidence energy loss to be a function of the square of both flow rate $Q$ and runner angular velocity $\omega$. Albuquerque et al. [8] suggest a range for $\lambda$ of $0.5-0.7$ and use 0.5 . For the laboratory-scale system, the higher value of 0.7 is chosen to reflect the use of non-optimal runner geometry. 


\subsubsection{Specific swirl energy loss}

Specific swirl energy loss is modelled as being proportional to the square of the tangential component of the exit velocity $V_{2 t}$ $[8]$,

$$
Y_{\text {LSwirl }}=\frac{1}{2} \chi\left(V_{2 t}\right)^{2},
$$

which can be written as

$$
Y_{\text {Lswirl }}=\frac{1}{2} \chi\left(r \omega-\frac{Q}{A_{d}} \cdot \tan \left(\beta_{2}-\delta\right)\right)^{2} .
$$

Albuquerque et al. [8] suggest a range for $\chi$ of $0.2-0.4$ and use 0.4 ; a mid-range value of 0.3 is adopted here.

\subsubsection{Calculating power loss from specific energy loss}

Power loss $P_{L}$ for each of the runner associated loss groups is calculated by integrating the product of the specific energy loss term $Y_{L}$ and the mass flow rate, across the runner swept area

$$
P_{L}=\int_{r_{\text {hub }}}^{r_{\text {tip }}} Y_{L} \cdot \frac{Q}{A_{d}} \rho \cdot 2 \pi r \cdot d r,
$$

noting that geometric parameters (e.g. $\beta_{2}$ and $\beta_{1}$ ), as well as flow variables (e.g. deviation $\delta$ ), are functions of $r$. In this way, all the terms in equation (4.2) can now be derived.

\subsubsection{Deriving drag torque from specific energy loss}

In an annulus of radius $r$ and width $d r$, perpendicular to the symmetry axis, the power lost can be written as

$$
d P_{L}=Y_{L} \cdot \frac{Q}{A_{d}} \rho \cdot 2 \pi r \cdot d r .
$$

Assuming that this power loss is concentrated in a specific region of the blade profile where the flow velocity is $V_{L}$, then the force imparted on the flow is

$$
d F_{L}=\frac{d P_{L}}{V_{L}}
$$

in the direction of the flow. The incremental torque is therefore the radial distance times the tangential component of this force

$$
d T_{L}=d F_{L} \cdot \sin \beta_{L} \cdot r .
$$

As profile loss is evaluated at the trailing edge [15, p.85] where the flow angle is $\beta_{2 f}$, the drag becomes

$$
T_{\text {Lprof }}=\int_{r_{\text {hub }}}^{r_{\text {tip }}} \frac{1}{W_{2}} \cdot Y_{\text {Lprof }} \cdot \frac{Q \rho}{A_{d}} \cdot 2 \pi r \cdot \sin \beta_{2 f} \cdot r d r,
$$

and similarly the drag due to incidence at the leading edge is

$$
T_{\text {Linc }}=\int_{r_{\text {hub }}}^{r_{\text {tip }}} \frac{1}{W_{1}} \cdot Y_{\text {Linc }} \cdot \frac{Q \rho}{A_{d}} \cdot 2 \pi r \cdot \sin \beta_{f 1} \cdot r d r .
$$

\subsection{Ductwork model}

The ductwork is the conduit that transfers water power from the river to the runner. The difference in elevation between the headwater and tailwater, gross head $H$, represents the energy per unit weight of fluid transferred from the flow when passing through the turbine. Not all of this energy is available to the runner, since ductwork head losses $h_{\text {duct }}$ are incurred due to work done overcoming viscous forces within the flow field and at solid boundaries,

$$
h_{\text {duct }}=H-h_{n} \text {. }
$$

The equation for ductwork head losses is dependent on whether the flow is developed or undeveloped. For smooth pipes, the distance from the inlet at which the turbulent boundary layer extends to the central axis is approximately 30 times the 
diameter, with the flow profile being considered fully developed from approximately 60 diameters [11, p.83]. The ductwork for the siphonic system described in Section 2 is a network of several sections, each with lengths typically smaller than three times their diameter. In addition, the constriction contributes to the reforming of a laminar boundary and a significant core region with a constant velocity profile [11, p.82]. Consequently, the flow can be considered undeveloped throughout the ductwork and head losses are approximately proportional to the square of the mean flow velocity [18]. The total ductwork head loss can be approximated as the sum of minor losses occurring within each section,

$$
h_{d u c t}=\sum_{i \text { sections }} h_{i}=\sum_{i \text { sections }} k_{i} \cdot \frac{\left(\frac{Q}{\bar{A}_{i}}\right)^{2}}{2 g},
$$

where $Q / \bar{A}_{i}$ is the average velocity within section $i, \bar{A}_{i}$ its mean cross-sectional area, and $k_{i}$ its geometry-dependent ductwork loss coefficient. Miller [11] empirically derives and tabulates loss coefficients for isolated components of numerous geometries, and specifies which area or velocity to apply. The following sections of the siphon turbine system are considered: inlet, straight, mitred bend, contraction, diffuser, and exit. Miller also provides a correction factor for the diffuser-bend combination as a function of geometry.

For many bounded flow applications, dynamic similarity is satisfied by equivalence of Reynolds number [6, p.174]. However, for pipe flow at high Reynolds numbers, frictional effects tend to become independent of Reynolds number [16, p.268]. This has been confirmed through testing on the laboratory scale model, revealing that the variance on a single loss coefficient, derived for the whole ductwork network, was small between $2.0 \times 10^{4}$ and $10^{5}$. Furthermore, the variance approached zero for Reynolds numbers above $10^{5}$. For general turbine operation, the Reynolds numbers are estimated to be greater than 20,000, consequently the single loss coefficient is taken to be constant, as are the individual loss coefficients $k_{i}$. Using equation (5.2), the flow rate $Q$ can now be written as

$$
Q=\sqrt{\frac{2 g\left(H-h_{n}\right)}{\sum k_{i} / \bar{A}_{i}^{2}}},
$$

where relevant areas $\bar{A}_{i}$ and loss coefficients $k_{i}$ are derived using [11].

\subsection{Weir model}

The gross head $H$ is dictated by the geometry of the weir and river profile, as well as the flow rate of the river. To simplify the modelling process we assume that the gross head remains constant, which serves to stabilise the power output of the system and can be achieved within certain river flow limits through careful design of the weir and river engineering.

\section{PARAMETER DETERMINATION AND COMPONENT MODEL VALIDATION}

In the following sections the tests are described that are used to determine model parameters for the particular laboratoryscale system studied here.

\subsection{Generator and rectifier parameters}

Two generators are compared, as described in Section 2, a modified 3-phase generator (nominal $832 \mathrm{rpm} / \mathrm{V}$ ) and a $12 \mathrm{~V}$ brushed DC machine. The machine constant $K_{e}$ for each type is determined through an open-circuit spin test, the results of which are presented in Figure 10.

Figure 10: Measured machine constants $K_{e}$ for the 3-phase brushless generator and the brushed DC machine, and the rectified open-circuit output voltage of the 3-phase generator-fed diode rectifier.

The measured phase resistance $R_{a}$ of the modified 3-phase generator is approximately $1.4 \Omega$ (including cable and contact resistances), the series inductance $L_{S}$ is approximately $20 \mathrm{mH}$. It is important to maintain the generator temperature constant, as self-heating significantly reduces $K_{e}$. The Schottky diodes' rated operating point is a forward voltage of $0.5 \mathrm{~V}$ at $2 \mathrm{~A}$. An $I_{S}$ of $8.90 \mathrm{nA}$ is determined at this operating point using equation (2.6) and assuming a typical value for $n$ of unity. The characteristic rectified generator equations (2.7) and (2.9) thus become

$$
\begin{aligned}
V_{D C}= & \sqrt{6} \cdot 0.0167 \cdot \omega_{g}-\frac{3}{\pi} \cdot 0.020 \cdot \omega_{g} I_{D C} \\
& -2 \cdot 1.4 \cdot I_{D C}-2 \cdot 0.026 \cdot \ln \left(\frac{I_{D C}}{8.9 \times 10^{-9}}\right)
\end{aligned}
$$


and

$$
T_{g}=\frac{3 \sqrt{6}}{2 \pi} \cdot 0.0167 \cdot I_{D C}
$$

Figure 11 (left) shows good agreement between experimentally derived output characteristics and equation (6.1). At $750 \mathrm{rpm}$ the short circuit current is $0.49 \mathrm{~A}$, providing a maximum torque of around $1 \mathrm{mNm}$.

Figure 11: Left: Comparison of model and experimental results for a diode-rectified 3-phase generator. Right: Fitting of the model to experimental results for a brushed DC generator.

A similar characteristic is measured for the brushed DC machine, Figure 11 (right). The model output can be fitted to this experimental data obtained from the DC machine by selecting a rated 'diode' forward voltage of $0.2 \mathrm{~V}$ at $2 \mathrm{~A}, n=2, K_{e}=$ $0.02 \mathrm{~V} /(\mathrm{rad} / \mathrm{s})$, a series inductance of zero and a winding resistance of $0.15 \Omega$. The resulting generator equations are

$$
\begin{aligned}
V_{D C}= & 0.02 \cdot \omega-2 \cdot 0.15 \cdot I_{D C} \\
& -2 \cdot 0.026 \cdot 2 \cdot \ln \left(\frac{I_{D C}}{0.0352}\right)
\end{aligned}
$$

and

$$
T_{g}=0.0227 \cdot I_{D C} \cdot
$$

Here, the 'diode' term in equation (6.3) models the non-linear contact voltage drop associated with the machine's brushed commutator. The torque constant of DC machines is equal to $K_{e}$ [12, p.65], shown in Figure 10, hence equation (6.4). The maximum torque at $750 \mathrm{rpm}$ when short circuited is $77 \mathrm{mNm}$. The generator's short-circuit torque should exceed twice the turbine's design torque in order to attain generator efficiency above $50 \%$. Increasing the short-circuit torque implies reducing the series resistance, which increases the size and cost of the generator.

The high (over-specified) torque of the DC machine allows more of the speed range to be observed, and it has the advantage of allowing the application of a negative load voltage in order to drive the machine against the torque of the runner, thereby further increasing the speed range. From this point forwards the model and testing shall concern the DC machine only. For practical realisations however, the more reliable permanent brushless magnet machine is preferred; therefore model equations (2.7) and (2.9) should be used, and scaled in $R_{a}$ and $K_{e}$ to obtain the desired efficiency.

\subsection{Transmission and inertia parameters}

Ideally the mechanical system would be characterised across a wide load and speed range using a torque transducer. A simplified method is adopted here, which may be more practical for a hydraulics laboratory, but which does not thoroughly validate the belt drive transmission model.

The parameters for the total transmission loss torque $T_{L}$ are obtained using three tests. In Test 1 , the dry unloaded system is driven over a wide speed range in the absence of water using the DC generator. Measured machine current delivers machine torque, which is referred to the runner side to obtain loss torque as a function of speed under no load, see Figure 12 . This delivers $T_{0}=0.057$ and $C_{\omega}=0.00023$ for equation (3.2). In Test 2, the flooded system is tested under constant flow rate of $0.0131 \mathrm{~m}^{3} / \mathrm{s}$ and the runner speed varied by adjusting the generator current. Runner side generator torque $G \cdot T_{g}$ is inferred from measuring generator current. This test did not allow the runner to be stalled using the generator alone. Instead the stallpoint is extrapolated from the data in Figure 12 to be $0.215 \mathrm{Nm}$. In Test 3, the runner stall torque is measured using strain gauge scales and a lever, and found to be $0.537 \mathrm{Nm}$. The difference is the transmission loss torque $T_{L}$ at zero speed and relatively high load. The solution $\left(T_{L}=0.322 \mathrm{Nm}, G \cdot T_{g}=0.215 \mathrm{Nm}, \omega=0\right)$ substituted into equation (3.2) delivers $C_{T}=$ 1.23 ,

$$
T_{L}=0.057+1.23 \cdot\left(1.76 \cdot T_{g}\right)-0.00023 \cdot \omega
$$

The relatively high losses are put down to non-radial loading of the bearings and sealing arrangement at high torque levels, and to belt drive friction at high speed and low load.

Figure 12: Experimental results used to determine loss torque parameters.

This method is repeated at $0.0105 \mathrm{~m}^{3} / \mathrm{s}$, which provides an inferred $G \cdot T_{g}$ of $0.12 \mathrm{Nm}$, and a stall torque of $0.37 \mathrm{Nm}$, providing a new value for $C_{T}$ of 1.608. $C_{T}$ appears to be a strong function of the load; therefore a quadratic dependency on load torque is adopted so that the transmission loss torque model fits both flow rates, 


$$
\begin{aligned}
T_{L}= & 0.057+2.06 \cdot\left(1.76 \cdot T_{g}\right)- \\
& 3.86 \cdot\left(1.76 \cdot T_{g}\right)^{2}-0.00023 \cdot \omega .
\end{aligned}
$$

The moment of inertia $J_{\text {Total }}$ is estimated to be of the order of $0.05 \mathrm{kgm}^{2}$; its accuracy is not important here as the model is not aimed at simulating dynamic transients, which is expected to require accounting for the inertia of the flow.

\subsection{Runner parameters}

As previously stated, the runner is an un-modified, off-the-shelf propeller and has not been optimised for hydraulic efficiency. Therefore, the following details for blade number and hub-tip ratio are for reader reference only. Additional geometric parameters are provided as requirements of the mathematical model.

The use of four blades is commensurate with other tested designs for small-scale, axial-flow turbines, such as 5 blades for Singh and Nestmann [19] and 4 blades for Albuquerque et al. [8]. However, the hub-tip ratio of 0.15 is relatively small compared with 0.3 for Singh and Nestmann [19], 2.5 for Albuquerque et al. [8] and 0.6 for Alexander et al. [10]. A smaller hub-tip ratio reduces velocities through the runner annulus. This is beneficial for reducing velocity-dependant hydraulic loss modes and the risk of cavitation; however, there is a greater variance in tangential velocity along the blade length, necessitating a greater degree of twist to ensure appropriate blade entry angles [19]. Further work is required to establish the full effect of geometric parameters on these selection criteria.

Additional geometric parameters of the runner model are measured at eight radial positions along the blade length, see Figure 13.

Figure 13: Measured runner geometry (curved, non-twisted sheet metal blades) and polynomial approximations in $\mathrm{r}$.

These parameters are approximated by $3^{\text {rd }}$ or $4^{\text {th }}$ order polynomials in $r$, Figure 13 . It should be noted that due to the modelling requirement all angles are measured tangentially. As a result, even a planar blade has an effective camber, and entry and exit angles are a function of radial position $r$. Equation (4.10) for the deviation angle $\delta$ has a significant effect on torque and it is sensitive to tolerances on chords and angles, Therefore it is useful to inspect deviation and incidence along the blade for different runner speeds, see Figure 14. The figure shows the flow angles, as defined in Figure 9, which are obtained by plotting the flow angle equations of Section 3.5.3 for specific $Q$ and $\omega$ using the polynomial fits of Figure 13.

Figure 14: Deviation and flow angles along the curved, non-twisted sheet metal blade at three different runner speeds and constant flow rate. See text for details.

The precision of measurement (accuracy, number of radial positions and the order of polynomials) has been increased until the deviation at no point exceeds the value at which the flow turning angle $\epsilon$ is zero. It is apparent that at any given runner speed, sections of the blade are off-design. At $70 \mathrm{rad} / \mathrm{s}(668 \mathrm{rpm})$ the tips produce a negative absolute flow exit angle $\alpha_{f 2}$, implying an opposing torque at the tips where power is being transferred to the flow. This 'pump' region reduces the aggregate torque across the whole runner.

The parameters for the deviation equation (4.10) are established using the measured torque speed characteristic of Figure 15. In this experiment, flow rate is held constant and gross head decreases with runner speed.

Figure 15: Experimental and modelled runner output characteristic for two constant flow rates, and simulated torque components.

The simulated runner torque for $Q=0.0131 \mathrm{~m}^{3} / \mathrm{s}$ is fitted to the measured data by setting the deviation parameters to $c_{o p}=$ 3.3 and $c_{i}=0.07 . c_{o p}$ has been scaled to produce an agreement at the design point, and then $c_{i}$ is adjusted to reduce the stall torque and increase the free-running velocity. The runner model is now fully parameterised. The preceding analysis shows that relatively accurate deviation parameters can be derived by carrying out a simple stall torque and a free running test without the belt drive in place. Figure 15 shows that reasonable agreement is also achieved at the lower flow rate, thereby further validating the model.

Figure 15 shows two simulated Euler torque curves; one neglecting deviation, which can be interpreted as an upper limit on achievable torque; and a second including deviation, which demonstrates its significant effect. The difference between the latter curve and that of the runner torque constitutes the sum of the drag torque components. An additional trace has been added representing the resulting runner torque if deviation parameters are adopted from the advanced design of [8], $c_{o p}=$ 1.5 and $c_{i}=0$.

Figure 16 shows results from the same experiment, again at a fixed flow rate and variable head. The term gross power $P$ represents the total drop in water power across the system and is a function of the gross head, 


$$
P=H \cdot Q \cdot \rho g .
$$

Ductwork head loss is measured without the runner in place and is assumed constant for a given flow rate. Ductwork loss is deducted from the gross power $P$ inferred from measurement to provide the net power available to the runner, labelled $P_{n}$ (experiment). The electrical power output is measured, summed with the mechanical losses (Figure 12) and multiplied by the gear ratio to provide the 'measured' runner shaft power $P_{r}$.

Figure 16: Runner output characteristic under fixed flow rate. Modelling data (dashed lines) and data inferred from measurement (solid lines with markers).

The dashed lines in Figure 16 show the modelled runner losses and runner shaft power $P_{r}$. It can be seen that the sum of modelled runner power losses are in good agreement with the measured gross power minus ductwork losses, until the runner approaches free-running speed.

\subsection{Ductwork parameters}

Measurements without the runner in place confirm a near quadratic behaviour of head against flow, in keeping with the literature. Figure 17 shows the measured steady-state flow rate against gross head across the ductwork, and the modelling results obtained by evaluating loss coefficients for the particular geometry of the physical model. Note that the model slightly under-predicts the flow rate, possibly because of neglected beneficial interactions between ductwork sections. Of these, only the interaction of bend and draft tube, or diffuser, has been considered.

Figure 17: Measured and modelled ductwork flow rate against gross head. The test setup incorporated the bearing housing and support stays but without a runner in-situ.

Details of the loss coefficients are as follows. The entry is modelled as a partially chamfered pipe inlet which takes account of the following straight section, and provides a loss coefficient $k_{\text {inlet pipe }}$ of approximately 0.4 [11, p.56]. For the mitred bend $k_{\text {bend }}=0.41$ [11, p.19], for the contraction $k_{\text {contraction }}=0.05[5, \mathrm{p} .390]$ and for the diffuser $k_{\text {diffuser }}=0.35$ [11, p.26]. In order to obtain the combination correction factor $C_{b-d}=0.5$ for a diffuser following a bend [11, p.29], it is assumed that the contraction is a straight element. The loss coefficients for these two elements are divided by this correction factor. In accordance with the literature, mean flow velocity is used to determine the head loss across each ductwork section, except for the diffuser where the entry velocity is used [11, p.23]. Velocity head of the flow discharging into the tailwater is assumed to be lost in turbulent mixing, therefore $k_{\text {exit }}=1$.

\section{SYSTEM SOLUTION METHOD AND DISCUSSION OF RESULTS}

\subsection{Steady-state solution method}

Previous sections have derived a steady-state system model. This can be written as a set of eight simultaneous equations in eight variables $Q, h_{n}, \omega, T_{r}, T_{L}, T_{g}, I_{D C}$ and $V_{D C}$, whilst the gross head $H$ and output power $P_{\text {Load }}$ are considered as control parameters:

$$
\begin{gathered}
Q=C_{1} \sqrt{H-h_{n}} \\
h_{n}=\int_{A_{r}} f_{2}(Q, \omega, r) d r \\
T_{r}=\int_{A_{r}} f_{3}(Q, \omega, r) d r \\
0=T_{r}-T_{L}-C_{4} \cdot T_{g} \\
T_{L}=C_{50}+C_{51} \cdot T_{g}+C_{52} \cdot T_{g}^{2}+C_{53} \cdot \omega \\
T_{g}=C_{6} \cdot I_{D C} \\
V_{D C}=C_{70} \cdot \omega-C_{71} \cdot \omega I_{D C}-C_{72} \cdot I_{D C} \\
-C_{73} \cdot \ln \left(C_{74} \cdot I_{D C}\right) \\
I_{D C}=C_{8} \cdot \frac{P_{L o a d}}{V_{D C}} .
\end{gathered}
$$

Written in this form, the $C_{i}$ represent constants that can be derived from the above analysis and depend on both the 
electromechanical properties and the geometry of the ductwork. In contrast, the functions $f_{2}$ and $f_{3}$ depend crucially on the blade geometry via the blade polynomials given in Figure 13, which in turn depend on the blade radial position $r$. Note that equation (7.4) is the steady-state version of equation (3.1). Thus, given gross head $H$ and output power $P_{\text {Load }}$, we have to solve a non-linear system of eight algebraic equations in the eight unknown variables. Clearly, since several of the equations are linear, as part of the solution procedure several variables could be eliminated. Nevertheless the solution of such a parameterised system of equations would require the use of a root finding method, such as Newton-Raphson. Such a fully parameterised investigation using numerical continuation theory will form the subject of future work.

An alternative method, used here, is to implement a time-domain system model and to seek steady-state points of operation by the introduction of time dependence into equation (7.4), as in equation (3.1). An integrator derives $\omega$ from equation (3.1). Two additional relaxation elements are added to reduce the stiffness of the system: these are filters, or delay functions, described by the transfer function $1 /\left(1+\frac{s}{T}\right)$, where $T$ is the delay time constant. $10 \mathrm{~ms}$ provides enough relaxation for a numerical solution using Matlab. A first delay element is inserted into the signal path of flow rate $Q$ between the ductwork and runner models, Figure 5. A physical interpretation is that on a step change in driving head, the inertia of the flow prevents an instantaneous increase in flow rate. A second relaxation element is introduced into the current feedback $I_{D C}$ from the load, representing delays in the voltage control and those due to the charging of a converter output capacitor.

\subsection{Discussion of steady-state results}

Figure 18 shows how the electrical sub-system affects the global system operating point for several fixed values of gross head $H$. As the electrical output current $I_{D C}$ is varied, the electrical power output $P_{\text {Load }}$ is found. Experimental results are also plotted for the 3 lowest heads. At the highest head, measurements are only partial due to the flow rate limitation of the flume.

Figure 18: Comparison of the system model (solid line) with test data showing electrical output power against runner shaft speed at different values of gross head. Negative powers are a result of the interpolation of measurements at slightly different heads.

For a given head, the power curve's right-hand intersection with the zero power line corresponds to the 'open-circuit' point, where the generator delivers no current to the load, and thus generates no torque. All the runner shaft power is consumed as internal transmission loss. As the load current increases towards an optimum, moving to the left on the power curve, the point of maximum power and optimal shaft speed is reached. Beyond the optimum, the power drops off to zero again; here, the generator is short circuited and is applying zero voltage to the load. In short circuit, the generator torque is at its highest; not sufficient to completely stall the turbine, but high enough to maintain a relatively low shaft speed.

The agreement between experiment and model found in Figure 18 is highly encouraging. Notice that the trend towards a maximum power point is captured well, as is the dependence of short-circuit and open-circuit operating points on the electric load. Moreover, for $H>0.1 \mathrm{~m}$, the optimum and short-circuit runner speeds agree closely.

At heads of $0.14 \mathrm{~m}$ and lower, the model over-predicts the open-circuit speed as a direct result of the runner model underpredicting the losses of the runner close to the free-running speed, see Figure 16. At the lowest gross head of $0.1 \mathrm{~m}$, torques are very low, and additional mechanical effects have come into play to cause at least some of the discrepancy between test and simulation results. It should be noted that, due to the low voltages generated at the lowest head, contact resistances can affect the accuracy of results.

Figure 19 shows more detail of the operation at a gross head of $0.2 \mathrm{~m}$. The figure can be read by starting at the point of highest shaft speed $(89 \mathrm{rad} / \mathrm{s})$, the open-circuit point. Here, the flow rate through the turbine is at a maximum of approximately $16.3 \mathrm{l} / \mathrm{s}$ and the runner shaft power being absorbed by the transmission losses is $3 \mathrm{~W}$ (both curves relate to the left-hand y-axis). The runner shaft torque which opposes the transmission loss torque is $0.4 \mathrm{Nm}$ (right-hand y-axis). Increasing the load current increases the generator torque, thereby slowing the shaft speed and increasing the runner torque. The runner now induces a higher net head, largely due to increasing runner associated losses, and therefore the flow rate reduces.

Figure 19: Flow rate, runner shaft power and runner torque against runner shaft speed at a constant head $H=0.2 \mathrm{~m}$. See text for details.

By comparing the runner shaft power curve of Figure 19 with the electric output power curve of Figure 18 at $H=0.2 \mathrm{~m}$, it can be seen that the points of maximum power for both runner shaft power and electric output power occur at slightly different speeds. A decrease in power towards lower speeds implies that for power or voltage regulated loads, the system is only stable down to around $60 \mathrm{rad} / \mathrm{s}$ at $H=0.2 \mathrm{~m}$. Raising the power demand at this speed stalls the turbine. An ohmic load provides stability over the whole speed range. Grid-tie converters can be designed to have either characteristic. The comparison of the two power curves also shows that a power of up to $9 \mathrm{~W}$ is absorbed by the transmission. This loss stems from non-ideal seals and bearings, a suboptimal belt-drive transmission and a DC generator, that, similar to passive rectification at low voltages, incurs a low efficiency. 
From Figure 19 the specific speed $N_{S}$ of the turbine can be derived. Substituting maximum power point values $(0.0102 \mathrm{~kW}$ and $51.2 \mathrm{rad} / \mathrm{s}$ at $0.2 \mathrm{~m}$ head) into equation (4.1) provides a specific speed of $38.7 \mathrm{rad} / \mathrm{s}$ or $369 \mathrm{rev} / \mathrm{min}$, which lies in the expected range for axial-flow turbines.

\section{CONCLUSIONS AND FURTHER WORK}

The main achievement of this work has been to derive and validate a complete model for a siphon turbine and generator system. The model takes as inputs axial-flow turbine geometry, ductwork geometry and electric parameters. The flow rate through the turbine is determined from the gross head across the system and the net head dropped across the runner. Runner torque has been calculated using the classic Euler torque equation, reduced by a conceptual model for drag torque mechanisms. A transmission model has been proposed and validated; demonstrating that transmission loss torque has a strong dependence on both runner speed and load torque, chiefly due to non-radial loading of bearings. The parameters for the model are obtained from the literature. Tuning of the model has been carried out only for two deviation related parameters one of which can be obtained from the literature for different types of turbine design and the other may be adopted from the work presented.

The complete system model enables power output prediction for varying runner speed, which is in turn determined from the load current drawn. From Figure 18 it can be seen that the correct trends are generated and that good agreement is obtained within the mid-range of gross head tested. Consequently, this model can be used in the conceptual design of axial-flow turbines and for correct generator sizing.

The ability to predict off-design performance means that the model can be used in conjunction with a river flow hydrograph or flow duration curve in order to produce estimates for annual energy yield. This information would be particularly useful in establishing the business case for a new hydropower scheme.

Prediction of off-design performance has been achieved through modification of Carter and Hughes' [15, p.80] original deviation equation. Non-zero angles of incidence, incurred as runner speed increases or decreases about the design point, are assumed to contribute to the flow turning angle at the runner inlet. In the case of under-speed, increasing incidence gives increasing deviation, thereby limiting the tangential component of axial flow. The result is that the semi-empirical equation, derived from blade testing with negligible incidence, can be used to calculate torque at off-design conditions, see Figure 15.

However, it is important to recognise the limitations of this approach in that it necessitates a test, albeit simple, in order to validate an additional coefficient $c_{i}$ as well as the over-prediction factor $c_{o p}$ suggested by Albuquerque. This highlights an opportunity to develop a dimensionless, incidence-dependant deviation model for hydropower runner blades. In order to improve this model, deviation at high speeds should become negative soon after the absolute flow exit angle $\alpha_{f 2}$ becomes negative, indicating pump behaviour (see Figure 14).

More generally, the overall form of equations (7.1) - (7.8) provides a general framework for modelling the operation of any siphonic hydropower system. In future, the form of these equations will be investigated more thoroughly to ascertain the sensitivity of each of the components of the model to the constants $C_{i}$ and functions $f_{2}$ and $f_{3}$ when expressed in dimensionless form. In addition, transient effects may be investigated through re-introduction of time-dependent terms into the equations. In so doing, we would hope to be able to recommend new guidelines for the design and operation of siphonic hydropower systems at domestic or commercial length scales.

A runner will incur a 'leakage loss' due to the clearance between the blade tips and the runner housing. The runner model incorporates a simplified allowance for leakage loss by using the runner housing and hub radii to calculate axial velocity, but integrating the torque balance between the hub and the tip radial stations. However, additional energy loss mechanisms occurring within the clearance region, and not accounted for by the profile loss model, are anticipated to result in an underestimation of the net head dropped across the runner and an overestimation of the flow rate.

The results of Figure 16 imply that the selected head loss mechanisms model the operation from stall to optimum speed reasonably well, but that towards the free-running speed the losses are underestimated. This may be due to an increasingly dominant loss mechanism not already accounted for. One example may be losses specifically attributed to blade tip vortices, although some account of tip related energy losses is reportedly made within Horlock's [15, p.85] profile loss model used here. However, no account has been taken of any additional drag torque mechanisms occurring within the clearance region. Combined with the assumed uniform axial velocity profile, this may be responsible for the overestimate of runner stall torque in Figure 15. Increased axial velocity owing to acceleration at the hub is also neglected; however, the hub only accounts for $2.4 \%$ of the runner area and if the acceleration is confined to the lower radial stations, influence on torque will be limited.

As a general remark, a more sophisticated representation of the loss and drag mechanisms within the clearance region may improve model accuracy for both stall torque and free-running head losses. In larger systems, the clearance would become a smaller fraction of the cross-section, thereby reducing the importance of an accurate representation of leakage. More suitable tip design would avoid the reduced chord which can be a source of modelling error, mitigated here by observing deviation 
along the length of the blade as a function of flow rate and runner speed (see Figure 14).

Loss coefficients tabulated by Miller [11] represent testing on components that are isolated by straight ductwork sections, the length of which depends on the type of section being isolated. Discounting the complex interactions between adjacent sections may have resulted in the small ductwork head loss error demonstrated in Figure 17. Minor losses due to the support stays at the bearing housing have also been neglected.

The system model proposed here has been validated through testing on a turbine with non-optimal runner geometry, incurring additional modelling complications due to variance in incidence and flow turning angle along the blade radius even at the design point, see Figure 14. It would be interesting to investigate the potential improvement in model accuracy, as well as efficiency, when using a bespoke runner design.

Finally, the scope of the system model requires extending to include a stator, or guidevanes. These are ubiquitously used in axial-flow turbines in order to induce a pre-swirl before the runner, thereby reducing swirl in the draft tube and the associated losses. A stator model would be similar to that of the runner, and would allow geometry optimisation of both components, resulting in the flow being axial on exit of the runner when operating at the design point. A direct drive should be envisaged, in order to avoid the high transmission losses incurred in the laboratory-scale system.

\section{ACKNOWLEDGEMENTS}

This project is funded by the Departments of Electrical \& Electronic Engineering and Engineering Mathematics of the University of Bristol, and supported by Motorola. The authors are grateful for the active technical support and advice from P. Bishop of Power Oasis; M. White of Motorola; H. Sharp, E. Matos, J. Loveless, A. Champneys, J. Smith, M. McCann, D. Drury, S. Williamson and J. F. Burn of the University of Bristol; and A. A. Williams of the University of Nottingham.

\section{LIST OF FIGURE CAPTIONS}

Figure 1: Schematic diagram of a siphon turbine installed over a weir with two paths for the water flow: over the weir and through the ductwork. The gross head is limited because the headwater can be raised only up to the weir height, the tailwater

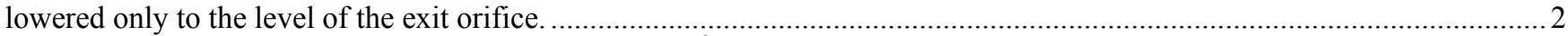

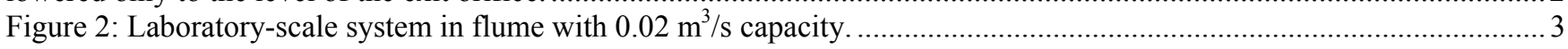

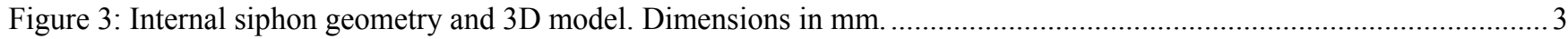
Figure 4: Un-modified, off-the-shelf propeller used as the runner for the laboratory-scale device. Blade angles, relative to the primary axis, increase from around $45^{\circ}$ at the hub to $60^{\circ}$ at the tip. The blades are slightly cambered. Details of the exact

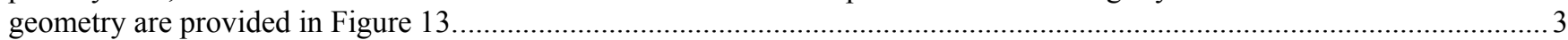
Figure 5: System model showing component input and output variables............................................................... 4 Figure 6: Schematic representation of the measurable input and output characteristics of the component models, with rough

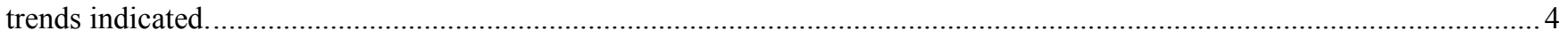
Figure 7: The generator and rectifier model represents a rectified 3-phase permanent magnet generator, represented here as a circuit diagram and time-domain voltage traces, see text for details. ................................................................. Figure 8: Runner model, represented as a block diagram showing component input and output variables. .......................... 6 Figure 9: Cross-section of an individual blade showing velocity triangles and notation used for runner model. ..................... 7 Figure 10: Measured machine constants $K e$ for the 3-phase brushless generator and the brushed DC machine, and the rectified open-circuit output voltage of the 3-phase generator-fed diode rectifier...................................................... 11 Figure 11: Left: Comparison of model and experimental results for a diode-rectified 3-phase generator. Right: Fitting of the

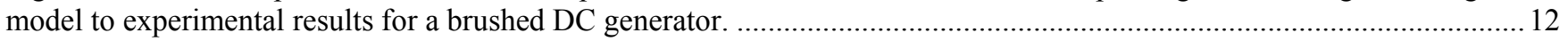
Figure 12: Experimental results used to determine loss torque parameters. ............................................................. 12 Figure 13: Measured runner geometry (curved, non-twisted sheet metal blades) and polynomial approximations in $\mathrm{r} . . . . . . . . .13$ Figure 14: Deviation and flow angles along the curved, non-twisted sheet metal blade at three different runner speeds and

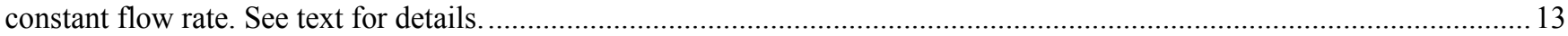
Figure 15: Experimental and modelled runner output characteristic for two constant flow rates, and simulated torque

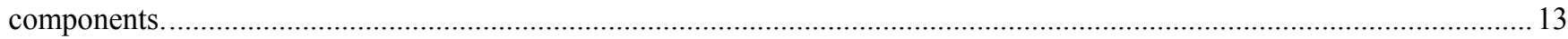
Figure 16: Runner output characteristic under fixed flow rate. Modelling data (dashed lines) and data inferred from

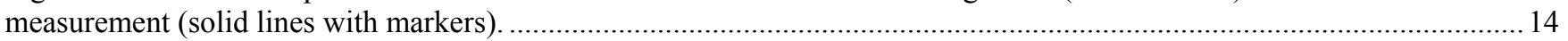
Figure 17: Measured and modelled ductwork flow rate against gross head. The test setup incorporated the bearing housing and support stays but without a runner in-situ.

Figure 18: Comparison of the system model (solid line) with test data showing electrical output power against runner shaft speed at different values of gross head. Negative powers are a result of the interpolation of measurements at slightly different heads.

Figure 19: Flow rate, runner shaft power and runner torque against runner shaft speed at a constant head $H=0.2 \mathrm{~m}$. See text for details. 


\section{FIGURES}

Supplied as separate tiff files. All figures are formatted to span one column of a two-column formatted paper.

\section{REFERENCES}

[1] O. Paish, Small hydro power: technology and current status, Renewable and Sustainable Energy Reviews, Volume 6, Issue 6, December 2002, pp. 537-556.

[2] P. Wiemann, G. Müller, J. Senior, Review of current developments in low head, small hydropower, 32nd IAHR Conference 2007, Venice, Italy, pp. 10 , Jul 2007.

[3] UK Environment Agency, Opportunity and Environmental Sensitivity Mapping for Hydropower in England and Wales, Bristol, February 2010.

[4] UK Department of Energy and Climate Change, Feed-in Tariffs - Government's Response to the Summer 2009 Consultation, London, February 2010.

[5] F.M. White, Fluid Mechanics, McGraw-Hill, $6^{\text {th }}$ edition, New York (1986).

[6] B.S. Massey, Mechanics of Fluids, $7^{\text {th }}$ Ed., Chapman \& Hall, New York (1998).

[7] K.V. Alexander, E.P. Giddens, Microhydro: Cost-effective, modular systems for low heads, Renewable Energy, Volume 33, Issue 6, June 2008, pp. 1379-1391.

[8] R. B. F. Albuquerque, N. Manzanares-Filho, W. Oliveira, Conceptual optimization of axial-flow hydraulic turbines with non-free vortex design, Proceedings of the Institution of Mechanical Engineers, Part A: Journal of Power and Energy, Volume 221, Number 5 / 2007.

[9] P. Singh, F. Nestmann, Exit blade geometry and part-load performance of small axial flow propeller turbines: An experimental investigation, Experimental Thermal and Fluid Science, Volume 34, Issue 6, September 2010, pp. 798-811.

[10] K.V. Alexander, E.P. Giddens, A.M. Fuller, Axial-flow turbines for low head microhydro systems, Renewable Energy, Volume 34, Issue 1, January 2009, pp. 35-47.

[11] D.S. Miller, Internal Flow: A Guide to Losses in Pipe and Duct Systems, Cranfield: British Hydromechanics Research Association, 1971.

[12] T. J. E. Miller, Brushless Permanent Magnet and Reluctance Motor Drives, Clarendon Press.

[13] N. Mohan, T.M. Underland, W.P. Robbins, Power Electronics, $3^{\text {rd }}$ ed., Wiley, New York, 2003.

[14] T. F. Chen, D.W. Lee, C. K. Sung, An Experimental Study on Transmission Efficiency of a Rubber V-Belt CVT, Mechanism and Machine Theory, Volume 33, Issue 4, pp. 351-363, May 1998.

[15] J. H. Horlock, Axial flow turbines, 1973 (Robert E. Krieger Publishing Company, Huntington).

[16] J.F. Douglas, J.M. Gasoirek and J.A. Swaffield (3rd ed.), Fluid Mechanics, Bath Press, Avon (1995).

[17] X. Qui, C. Mallikarachi, M. Anderson, A New Slip Factor Model for Axial and Radial Impellors, ASME Turbo-Expo, Canada 2007.

[18] B.R. Munson, Fundamentals of Fluid Mechanics, Wiley, 2006.

[19] P. Singh, F. Nestmann, Experimental optimization of a free vortex propeller runner for micro hydro application, Experimental Thermal and Fluid Science, Volume 33, Issue 6, September 2009, pp. 991-1002. 